\section{Weniger kutane Probleme bei Melanom-Kombitherapie}

\begin{abstract}
Wird ein BRAF-Hemmer mit einem MEK-Hemmer kombiniert, kommt es seltener zu schweren Hautkomplikationen als bei einer Monotherapie. Vor allem Plattenepithelkarzinome treten dann seltener auf.
\end{abstract}

$E^{s}$ dürfte in der Medizin eher selten sein, dass unter einer Monotherapie gegen ein bestimmtes Leiden häufiger Nebenwirkungen auftreten als unter einer Kombitherapie. Genau das ist aber der Fall, wenn man bei der Melanomtherapie einen BRAF- und einen MEKHemmer kombiniert. Zumindest die kutanen Nebenwirkungen sind dann offenbar weniger gravierend als bei einer BRAF-Hemmer-Monotherapie. Erklä- ren lässt sich dies über eine paradoxe Aktivierung des MAP-Kinase-Pfads in Keratinozyten ohne BRAF-Mutation. Eigentlich sollte dieser intrazelluläre Signalweg durch BRAF-Hemmer blockiert werden. Eine Kombination mit einem MEK-Hemmer, der an einer anderen Stelle des MAP-Kinase-Wegs angreift, erreicht dieses Ziel offenbar besser, wohingegen unter einer BRAF-Hemmer-Monotherapie Keratinozyten ver-

\title{
Seltene Komplikation von Kompressionsstrümpfen
}

Ein 87-jähriger Mann mit Diabetes, Hypertonie, koronarer Herzkrankheit und Symptomen einer peripheren arteriellen Verschlusskrankheit (pAVK) wurde wegen eines rechtsseitigen Kolonkarzinoms stationär zur Hemikolektomie aufgenommen. Gemäß den Vorgaben des Krankenhauses zur Prophylaxe thromboembolischer Ereignisse erhielt er niedermolekulares Heparin und Kompressionsstrümpfe. Postoperativ stellte man eine Nekrose an der Spitze der rechten Großzehe fest.

Patienten mit einem hohen Risiko für eine pAVK müssen vor Anpassung von Thrombosestrümpfen hinsichtlich ihrer Durchblutungsverhältnisse sorgfältig untersucht werden. Entsprechend der Leitlinie des National Institute for Health and Care Excellence aus dem Jahr 2010 sollten bei Vorliegen einer Durchblutungsstörung alternative Maßnahmen erwogen werden, zum Beispiel eine pneumatische Kompression oder Fuß-Impuls-Technologie.

Karim A et al. A rare complication of antiembolism stockings. BMJ 2015; 350: h1174

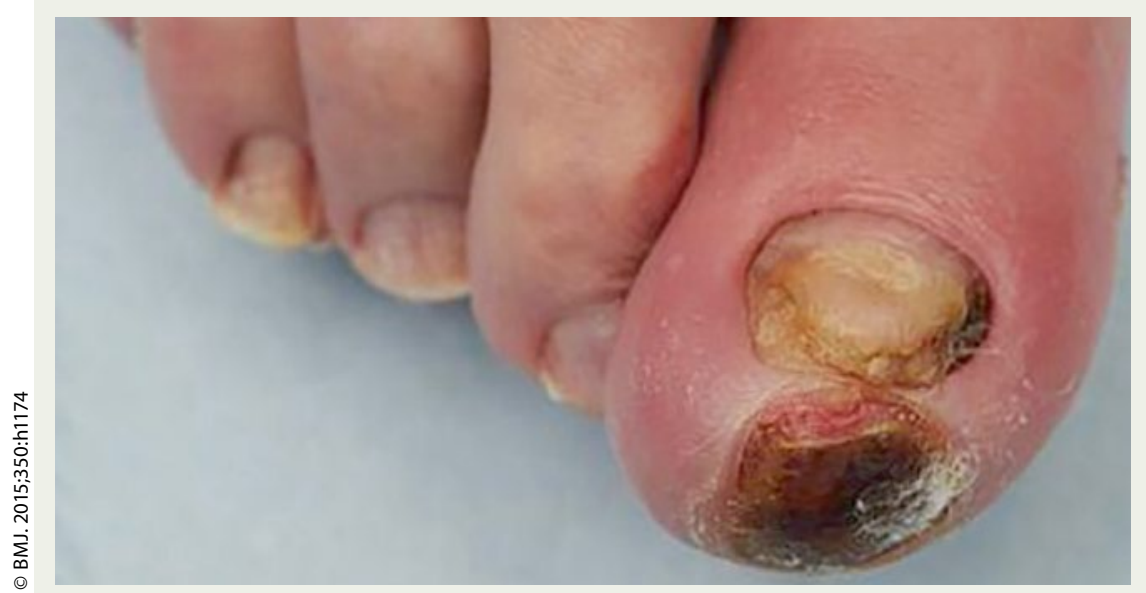

Nekrose an der Spitze der rechten Großzehe nach dem Tragen von Kompressionsstrümpfen im Krankenhaus

mehrt hyperproliferieren. Erste Hinweise auf kutane Vorteile der Kombitherapie hatte es bereits zuvor gegeben.

Dies können nun australische Dermatologen bestätigen, die sich 185 Patienten vor und nach der Behandlung mit BRAF-Hemmern genauer angeschaut haben. 119 davon hatten eine Monotherapie mit dem BRAF-Hemmer Dabrafenib bekommen, 36 eine mit Vemurafenib, und 30 waren mit Dabrafenib plus dem MEK-Hemmer Trametinib (CombiDT-Therapie) behandelt worden. Die Therapiedauer lag in allen drei Gruppen bei etwa einem halben Jahr.

Hauttoxische Effekte wurden bei fast allen Patienten (92\%) mit einer BRAFHemmer-Monotherapie und den meisten mit CombiDT (77\%) beobachtet. Allerdings unterschieden diese sich deutlich: Plattenepithelkarzinome wurden bei $26 \%$ der Patienten mit Dabrafenib und 36\% mit Vemurafenib beobachtet, aber bei keinem Patienten mit der Kombitherapie. Eine transitorische akantholytische Dermatose (GroverErkrankung) entwickelten rund $40 \%$ der Patienten mit BRAF-Hemmer-Monotherapie, aber keiner mit der Kombibehandlung. Die Patienten mit CombiDT blieben auch komplett von einer Keratosis verrucosa verschont, dagegen konnten Dermatologen diese Störung bei mehr als zwei Drittel der Patienten mit BRAF-Hemmer-Monotherapie nachweisen. Deutlich häufiger traten unter der Kombitherapie akneiforme Reaktionen (17\%), Follikulitis (40\%) und allgemeine Entzündungen auf (20\%). Bei den Monotherapien lagen die Raten hierfür im einstelligen Prozentbereich.

Fazit: Insgesamt entwickelten die Patienten mit der Kombitherapie deutlich seltener schwere und problematische kutane Nebenwirkungen. Da allerdings nur ein kleiner Teil der Melanomkranken per Kombitherapie behandelt worden war und zudem keine Randomisierung stattfand, sind die Resultate der australischen Studie nur bedingt aussagekräftig.

Thomas Müller

Carlos G et al. Cutaneous Toxic Effects of BRAF Inhibitors Alone and in Combination With MEK Inhibitors for Metastatic Melanoma. JAMA Dermatol 2015; 151: 1103-9 